\title{
Immunization in Special Situations
}

\author{
SANJIB Mondal and SurJIt Singh \\ Allergy Immunology Unit, Department of Pediatrics, Advanced Pediatrics Centre, \\ PGIMER, Chandigarh 160012, India.surjitsinghpgi@rediffmail.com
}

$\mathrm{P}$ rotection against vaccine preventable diseases (VPDs) constitutes one of the most effective ways to impact child health worldwide. The World Health Organisation (WHO) launched the Expanded Programme on Immunisation (EPI) in 1974 [1]. India implemented the EPI in 1978. The Universal Immunisation Programme was launched in 1985 with a focus on infants below the age of one, and included tetanus toxoid for pregnant women [2]. Over the last three decades, there have been major advances in coverage against VPDs in our country. As a result, there has been a significant reduction in occurrence of VPDs in India as also in other developing countries.

With improvements in provision of health care in our country, an increasing number of children with serious medical disorders are now surviving childhood and growing up to be adults. Many amongst these medical disorders also have a component of secondary immunodeficiency, as for instance $\beta$ - thalassemia major, nephrotic syndrome, chronic renal failure and chronic liver disease, and human immunodeficiency virus (HIV) infection.

While there have been several published studies of immune responses following immunization in children with primary immune deficiencies [3], there is a paucity of literature on immune protection in patients with secondary immunodeficiencies. In this issue of the journal, there are three studies that fill up some of these lacunae in the literature [4-6].

Gomber, et al. [4] have determined anti-HBs antibody levels in 85 multi-transfused children with $\beta$-thalassemia major who had received primary hepatitis $B$ vaccination at least 5 years ago. The authors report that seroprotection rates of hepatitis $\mathrm{B}$ vaccine after a mean duration of 10.8 years of completion of primary immunization were significantly higher amongst children with $\beta$-thalassemia major compared to healthy controls. Authors also found that the 23 seronegative children with $\beta$-thalassemia major were able to achieve adequate seroprotection after a single booster dose of hepatitis B vaccine. There are two important messages in this study viz., i) majority of multi- transfused children with $\beta$-thalassemia major had adequate seroprotection titers even after 5 years of primary vaccination, and ii) following a single booster dose, children who were seronegative were able to mount an adequate protective response to the vaccine.

This study suggests that regular assessment of antiHBs titres following primary hepatitis $B$ vaccination needs to be incorporated in the management protocol of this condition. This is important because it is known that children with multi-transfused $\beta$-thalassemia major are at high risk of acquiring hepatitis $B$ infection. Though the numbers recruited in this study are admittedly small, it needs to be noted that this is a single center study and there were time constraints for completion. Nevertheless, the results are likely to impact management of children with $\beta$ thalassemia major in our country.

Jain, et al. [5] report a randomized control trial in children with HIV infection on antiretroviral therapy (ART) that compared seroprotection rate and anti-HBs titres following primary immunization with double strength $(20 \mu \mathrm{g})$ recombinant hepatitis B vaccination. The authors compared two vaccination schedules- 3 dose $(0,1$ and 6 months) vs 4 dose ( $0,1,2$ and 6 months). Data on an accelerated 3 dose schedule ( 0,1 and 2 months) within the 4 dose group were analyzed separately. Study sample consisted of HIV infected children aged 18 months- 12 years who had received at least 6-months ART, had not received any previous dose of hepatitis $B$ vaccine and were anti-HBs negative. Authors found that while the median anti-HBs titres at 7 months were significantly higher in children who had received 4 doses, the difference had plateaued down by 12 months. Further, the accelerated 3 dose schedule resulted in comparable anti-HBs titers when compared to the conventional three dose schedule.

In this elegant study [5], it has been shown that the three dose double strength recombinant hepatitis B vaccination schedule offers comparable seroprotection to a four dose schedule for HIV infected children receiving ART. However, we need more data on long-term immunogenicity of the three dose accelerated schedule. This study 
needs to be replicated on a much larger sample size before results can be extrapolated for formulation of immunization policies for the country at large.

Nephrotic syndrome is one of the commonest chronic renal disorders in children. Children with nephrotic syndrome have a compromised immune system both because of primary disease as well as the accompanying treatment with immunosuppressant agents. However, there are hardly any data on seroprotection for VPDs from our country. While there have been some studies suggesting that antibody titres for common VPDs (such as diphtheria, pertussis, tetanus and measles) are lower in children with steroid sensitive nephrotic syndrome (SSNS), there is paucity of similar information in children with steroid resistant nephrotic syndrome (SRNS). Ajay, et al. [6] enrolled 76 children with nephrotic syndrome to determine seroprotective titers for diphtheria, pertussis, tetanus and measles after primary immunization. Forty amongst these had SSNS while 36 had SRNS. Authors found that seroprotection rates for diphtheria, pertussis, tetanus and measles were lower in patients with SRNS compared to SSNS. These findings appear to be in consonance with a previous study from the same centre [7], wherein it was shown that seroprotection rates of hepatitis $B$ vaccine in SRNS were lower when compared to SSNS.

Authors have clearly shown that children with nephrotic syndrome had lower overall seroprotective titers against diphtheria, pertussis, tetanus and measles even during periods of disease remission [6]. Further, the seroprotection was lower in those with SRNS. Authors recommend a booster dose of DTP or Tdap (in children above 7 years) and an additional dose of MR/MMR for all children with nephrotic syndrome once the child is in remission.
All three studies [4-6] have the apparent lacuna of a small sample size. However, this is understandable as the inclusion criteria were well-defined and rather restrictive. These studies have been well conducted and results are of direct clinical relevance to pediatricians in India. We need more data on protection against VPDs in children with secondary immunodeficiencies from our country, and such studies are a step in the right direction.

Funding: None; Competing interests: None stated.

\section{REFERENCES}

1. Government of India. Multi Year strategic Plan for Universal Immunization Program in India (2005-2010). Ministry of Health and Family Welfare; 2005.

2. UNICEF. World declaration on the Survival production and development of children: The challenge. World Summit for Children, 1990. Accessed on 5 February, 2021. Available from: http://www.unicef.org/wsc/declare.htm\# Thechallenge

3. Bonilla FA. Vaccines in patients with primary immune deficiency. Immunol Allergy Clin North Am. 2020;40: 421-35.

4. Gomber S, Yadav R, Dewan P, Ramachandran VG, Puri AS. Requirement of a booster dose of hepatitis $B$ vaccine in children with thalassemia 5 years after primary vaccination. Indian Pediatr. 2021;58:237-40.

5. Jain P, Dewan P, Gomber S, Kashyap B, Raizada A. Three vs four dose schedule of double strength recombinant hepatitis-b vaccine in HIV-infected children: a randomized controlled trial. Indian Pediatr. 2021;58:224-28.

6. Ajay M, Mantan M, Dabas A, Asraf A, Yadav S, Chakravarti A. Seroprotection for diphtheria, pertussis, tetanus and measles in children with nephrotic syndrome. Indian Pediatr. 2021;58:233-36.

7. Mantan M, Pandharikar N, Yadav S, Chakravarti A, Sethi GR. Sero-protection for hepatitis B in children with nephrotic syndrome. Pediatr Nephrol. 2013;28:2125-30. 